\title{
Facile Preparation of Water-Soluble $N$-Acetylated Chitosan and Molecular Weight Dependence of Its Water-Solubility
}

\author{
Naoji KubOTA ${ }^{\dagger}$ and Yukari EgUCHI \\ Department of Applied Chemistry, Faculty of Engineering, Oita University, \\ Dannoharu, Oita 870-11, Japan
}

(Received July 4, 1996)

\begin{abstract}
A simple procedure to prepare water-soluble chitosan (partially $N$-acetylated chitosan) was developed, and the water-solubility of $\mathrm{N}$-acetylated chitosan was investigated in detail, preparing a series of chitosan samples with various molecular weights. To reduce the molecular weight, chitosan was treated with $\mathrm{NaBO}_{3}$ under a homogeneous condition where chitosan was previously dissolved in an aqueous acetic acid. Weight-average molecular weights of chitosan samples obtained were determined by means of GPC equipped with a low angle laser light scattering photometer. Then each chitosan sample was $N$-acetylated with acetic anhydride under a homogeneous condition where chitosan was dissolved in a simple aqueous acetic acid. The degree of $N$-acetylation was estimated by ${ }^{1} \mathrm{H}$ NMR spectroscopy. As a result, the water-solubility of $N$ acetylated chitosan with about $50 \%$ substitution increased as the molecular weight decreased. The sequence of $\mathrm{N}$-acetylglucosamine and glucosamine residues was also determined by ${ }^{1} \mathrm{H}$ NMR spectroscopy.

KEY WORDS Chitin / $N$-Acetylated Chitosan / Water-Solubility / Molecular Weight / Gel Permeation Chromatography / Degree of $\mathrm{N}$-Acetylation / ${ }^{1} \mathbf{H}$ Nuclear Magnetic Resonance Spectroscopy /
\end{abstract}

Chitin, poly- $\beta(1 \rightarrow 4)-N$-acetyl-D-glucosamine, is one of the most abundant natural polysaccharides and available largely in the exoskeletons of shellfish and insects. It is obtained primarily as a by-product of seafood industry. Deacetylation of chitin readily affords chitosan, poly$\beta(1 \rightarrow 4)$-D-glucosamine. Chitin, chitosan and their derivatives have recently found a wide variety of applications as functional materials. ${ }^{1}$ We have already reported on the membrane applications of chitosan and its derivatives. ${ }^{2-4}$ However, the lack of solubility of chitin limits the practical usefulness. Since chitosan has amino group in the repeating unit, it is soluble in aqueous acidic media, but inherently water-insoluble. A considerable amount of research has been conducted in methods to render chitosan water soluble, e.g., changing polymer morphology and molecular weight, ${ }^{5}$ and formation of microcrystalline chitosan. ${ }^{6}$ In addition, chemical modifications can make chitosan water-soluble, depending on the molar substitution levels. Sannan et al. reported that, by a treatment of chitin with alkali under homogeneous condition, chitin with about $50 \%$ deacetylation became water-soluble. ${ }^{7,8}$ They suggested that the solubility arose from the randomly distributed acetyl groups at half the number of amino groups. Kurita et al. also prepared water-soluble chitosan with about $50 \% \mathrm{~N}$-acetylation from chitosan. ${ }^{9}$ However, very long reaction time was needed in the former case, and the complex solvent system, aqueous acetic acid/ methanol/pyridine, was used in the latter case. On the other hand, Aiba concluded that reduction of the molecular weight did not affect the water-stability of partially $N$-acetylated chitosan. ${ }^{10}$ However, clear relationship between molecular weight and water-solubility was not indicated; he used only two kinds of molecular weights: $10^{6}$ and $10^{5}$.

In this paper, we report an improved method to prepare water-soluble chitosan (partially $N$-acetylated chitosan). Chitosan is preliminary degraded by treatment

\footnotetext{
$\dagger$ To whom correspondence should be addressed.
}

with $\mathrm{NaBO}_{3},{ }^{11}$ and then each product is $\mathrm{N}$-acetylated with acetic anhydride in a simple aqueous acetic acid as solvent. The dependence of the water-solubility on the $N$-acetylation degree and the molecular weight is investigated quantitatively. Furthermore, the composition and the sequence of $N$-acetylglucosamine (GlcNAc) and glucosamine $(\mathrm{GlcN})$ residues are determined by ${ }^{1} \mathrm{H}$ NMR spectroscopy. NMR method is the most effective technique to determine concurrently the degree and the position of substitution of chitosan derivatives. ${ }^{12}$

\section{EXPERIMENTAL}

\section{Materials}

Chitosan is a commercial material purchased from Tokyo Kasei Kogyo Co., Ltd., and purified before use; chitosan was dissolved in 5\% aqueous acetic acid, filtered with a sintered glass filter, precipitated with $1 \mathrm{~mol} \mathrm{dm}^{-3}$ $\mathrm{KOH}$ ( $\mathrm{pH} 10-11$ ), rinsed with deionized water until neutral. This purification procedure was repeated three times, then it was washed with acetone and dried in vacuo. Standard pullulans for GPC were purchased from Showa Denko, Co., Ltd. Acetic anhydride, acetone, and methanol were distilled just before use. All other chemicals were of reagent grade and used without further purification.

\section{Degradation of Chitosan}

Purified chitosan $(0.5 \mathrm{~g})$ was dissolved in $5 \%$ aqueous acetic acid $\left(30 \mathrm{~cm}^{3}\right)$ in a round bottom flask, then aqueous $\mathrm{NaBO}_{3}\left(100 \mathrm{~cm}^{3}\right)$ of a desired concentration was added. After stirring at predetermined temperature and for predetermined time, the reaction mixture was cooled and $1 \mathrm{~mol} \mathrm{dm}^{-3} \mathrm{KOH}$ solution was added up to $\mathrm{pH} 10-11$. The obtained precipitate was centrifuged, and washed well with deionized water until neutral. Finally, it was washed with acetone and dried in vacuo at room temperature, and further dried under vacuum at $50^{\circ} \mathrm{C}$. IR spectra were taken with $\mathrm{KBr}$ pellets on a Hitachi 270-50 IR spectrophotometer. 


\section{Determination of Molecular Weight}

Weight-average molecular weights of the degraded chitosan samples were determined with a Tosoh GPC system (pump, CCPS; degasser, SD-8022; column, TSKgel GMPW XL ; column heater, U-620 VP30) equipped with a low angle laser light scattering photometer (LS-8000) and a differential refractometer (RI8011). Each sample was dissolved in $1.0 \mathrm{~mol} \mathrm{dm}^{-3}$ acetate buffer, which was the eluent, and filtered with a microfilter $(0.45 \mu \mathrm{m})$, and $100 \mathrm{~mm}^{3}$ of the sample solution was injected. The flow rate was $1.0 \mathrm{~cm}^{3} \mathrm{~min}^{-1}$, and column temperature was $40^{\circ} \mathrm{C}$.

\section{$N$-Acetylation of Chitosan}

Chitosan $(0.3 \mathrm{~g})$ was dissolved in $10 \%$ aqueous acetic acid $\left(50 \mathrm{~cm}^{3}\right)$, and desired amount of acetic anhydride was added. After stirring at ambient temperature for $5 \mathrm{~h}$, $5 \mathrm{~mol} \mathrm{dm}^{-3} \mathrm{NaOH}$ was added up to $\mathrm{pH} 8-9$ to stop the reaction. The reaction mixture was then dialyzed against pure water for $2 \mathrm{~d}$ to remove microions, and freeze-dried. The acetylated chitosan was treated with $1 \mathrm{~mol} \mathrm{dm}^{-3}$ methanolic $\mathrm{KOH}$ at room temperature for $5 \mathrm{~h}$. After alkaline treatment, $N$-acetylated chitosan was washed with methanol repeatedly using a centrifuge. At last, it was dissolved in deionized water and freeze-dried.

\section{Estimation of Water-Solubility}

$\mathrm{N}$-Acetylated chitosan samples $(50 \mathrm{mg})$ were dissolved in deionized water $\left(5 \mathrm{~cm}^{3}\right)$, and the transmittance of the solution at $600 \mathrm{~nm}$ was recorded on a Hitachi U-1000 spectrophotometer using a quartz cell with optical path length of $1 \mathrm{~cm}$.

\section{NMR Spectroscopy}

${ }^{1} \mathrm{H}$ NMR spectra were recorded on a Bruker ARX 300 spectrometer at $25^{\circ} \mathrm{C}$. $N$-Acetylated chitosan samples were twice freeze-dried from $\mathrm{D}_{2} \mathrm{O}(>99.75 \%)$ solutions to minimize the HOD signal. All samples were dissolved in $5 \mathrm{~mm}$ diameter tubes, and the sample concentrations were $20 \mathrm{mg} \mathrm{cm}^{-3}$. For water-insoluble samples, $\mathrm{CF}_{3} \mathrm{COOD}(>99.5 \%)$ was added. The chemical shifts were given on the $\delta$ scale relative to external TMS reference. The measurement conditions were as follows: a spectral window of $8064 \mathrm{~Hz}$, data points of $32 \mathrm{k}$, a pulse width of $3 \mu \mathrm{s}$, an acquisition time of $2.03 \mathrm{~s}$, and 32 scans with delay of $1 \mathrm{~s}$ between scans.

The 2D ${ }^{1} \mathrm{H}-{ }^{1} \mathrm{H}$ chemical shift correlation spectra (COSY) were obtained using $2048 \times 256$ data matrix size, zerofilled in the $F_{1}$ direction and symmetrized about the diagonal. Spectral widths were $3300 \mathrm{~Hz}$ in $F_{2}$ and $F_{1}$ dimensions, and 8 scans were recorded for each of 256 experiments with delay of $2 \mathrm{~s}$ between scans.

In order to diminish the line width of the anomeric proton signals, each $N$-acetylated chitosan sample with about $50 \%$ acetylation was dissolved in $\mathrm{D}_{2} \mathrm{O}$ containing $\mathrm{DCl}(>99.5 \%)$, and ${ }^{1} \mathrm{H}$ NMR spectra were recorded at $80^{\circ} \mathrm{C}$.

\section{RESULTS AND DISCUSSION}

A number of reports concerning the degradation of chitosan are available; $\mathrm{NaBO}_{3}$ was adopted in this study. ${ }^{11}$ To promote the reaction under a homogeneous condition, aqueous acetic acid was used as solvent in which chitosan was dissolved completely. Figure 1 shows IR spectra of chitosan samples before and after degradation. As can be seen, there is no significant difference between them. Table I summarizes the dependence of the weight-average molecular weight on the degradation conditions, along with the $M_{w} / M_{n}$ ratios determined by means of GPC. Chitosan was degraded over the molecular weight range from 600000 to 8800 . It is strongly dependent on the $\mathrm{NaBO}_{3}$ concentration, reaction temperature, and reaction time. Figure 2 exhibits the differential molecular weight distributions of degraded chitosan samples. The $M_{w} / M_{n}$ ratio of original chitosan is 2.53 and degraded chitosan samples have almost the same value: $2.2-2.5$. The chitosan main chain was thought to be cleaved randomly.

Each degraded sample thus obtained was acetylated with acetic anhydride. The reaction should be conducted under a homogeneous condition where chitosan is dissolved throughout. As solvent, simple aqueous acetic acid was used in this study. Since the reaction mixture showed a homogeneity during the reaction, the acetyla-

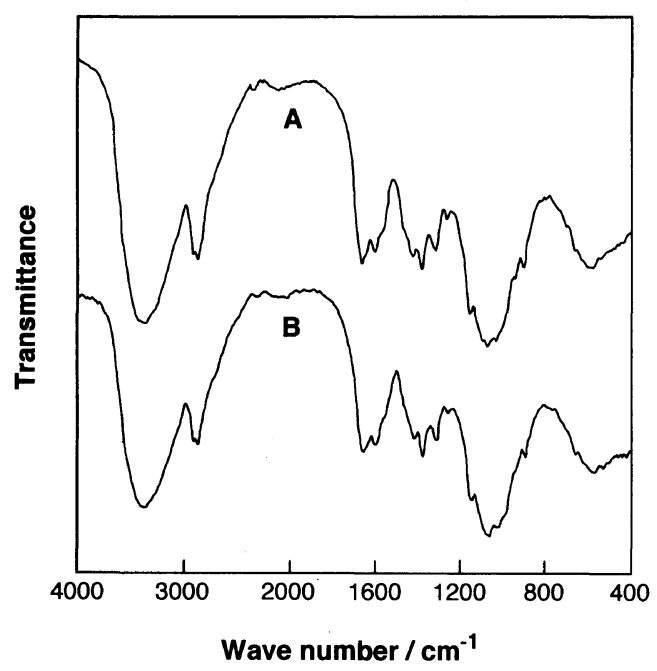

Figure 1. IR spectra of original and degraded chitosan samples: A, original chitosan; $\mathrm{B}$, degraded chitosan with sat. $\mathrm{NaBO}_{3}$ at $50^{\circ} \mathrm{C}$ for $2 \mathrm{~h}$ (see Table I).

Table I. Dependence of weight-average molecular weight on degradation conditions

\begin{tabular}{|c|c|c|c|c|c|}
\hline \multirow{2}{*}{$\begin{array}{l}\text { Sample } \\
\text { code }\end{array}$} & $\begin{array}{c}\mathrm{NaBO}_{3} \\
\text { concentration }\end{array}$ & Temp & Time & $M_{w}^{\mathrm{a}}$ & \multirow{2}{*}{$M_{w} / M_{n}$} \\
\hline & $\mathrm{w} / \mathrm{v} \%$ & ${ }^{\circ} \mathrm{C}$ & $\mathrm{h}$ & $\times 10^{4}$ & \\
\hline Ch-600 & Untreated & & & 60 & 2.53 \\
\hline Ch-380 & 0.01 & r.t. & 1 & 38 & 2.48 \\
\hline Ch-340 & 0.01 & 30 & 1 & 34 & 2.29 \\
\hline $\mathrm{Ch}-230$ & 0.01 & 50 & 1 & 23 & 2.36 \\
\hline Ch-150 & 0.05 & 50 & 1 & 15 & 2.29 \\
\hline Ch-140 & 0.2 & 50 & 1 & 14 & 2.33 \\
\hline $\mathrm{Ch}-110$ & 0.5 & 50 & 1 & 11 & 2.15 \\
\hline $\mathrm{Ch}-78$ & 1.0 & 50 & 1 & 7.8 & 2.36 \\
\hline Ch-63 & 2.0 & 50 & 1 & 6.3 & 2.28 \\
\hline Ch-33 & Sat. & 50 & 1 & 3.3 & 2.42 \\
\hline Ch-19 & Sat. & 50 & 2 & 1.9 & 2.25 \\
\hline Ch-12 & Sat. & 50 & 4 & 1.2 & 2.46 \\
\hline Ch- 8.8 & Sat. & 50 & 8 & 0.88 & 2.35 \\
\hline
\end{tabular}

${ }^{a}$ Weight-average molecular weight determined by GPC. 


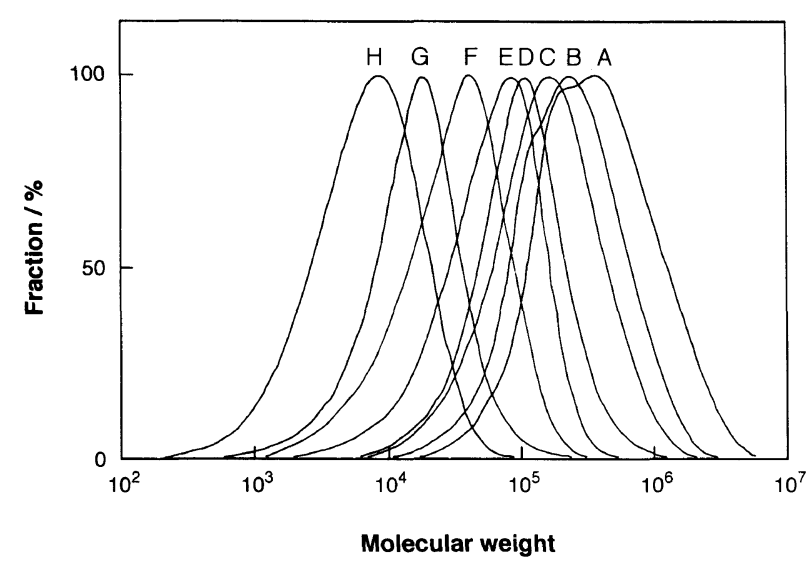

Figure 2. Differential molecular weight distribution curves for original and degraded chitosan samples: A, Ch-600; B, Ch-340; C, Ch-230; D, Ch-150; E, Ch-78; F, Ch-33; G, Ch-19; H, Ch-8.8. Sample codes correspond to those in Table I.

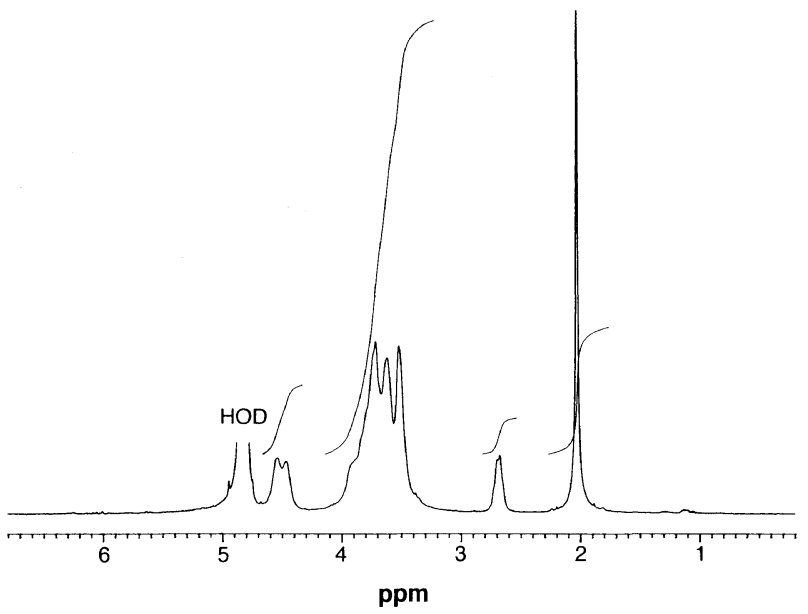

Figure 3. ${ }^{1} \mathrm{H}$ NMR spectrum of partially $N$-acetylated chitosan in $\mathrm{D}_{2} \mathrm{O}$. The sample was derived from Ch-19.

tion seemed to proceed randomly. The acetylation appeared to be quite selective, preferring the more nucleophilic glucosamine nitrogen, and we did not expect the formation of $O$-acetylated derivative in such an aqueous medium. However, additional treatment with methanolic $\mathrm{KOH}^{13}$ was carried out to make assurance doubly sure.

Figure 3 depicts the ${ }^{1} \mathrm{H}$ NMR spectrum of partially $N$-acetylated chitosan in $\mathrm{D}_{2} \mathrm{O}$. The spectrum gives intricate signals due to the copolymer of $\mathrm{N}$-acetylglucosamine and glucosamine. The resonance at $2.04 \mathrm{ppm}$ is easily assigned to the three $N$-acetyl protons. The other assignments are solved using $2 \mathrm{D}{ }^{1} \mathrm{H}-{ }^{1} \mathrm{H}$ chemical shift correlations shown in Figure 4. The $\mathrm{H}-1$ proton is directly assigned as the lowest peak; from it, the correlated $\mathrm{H}-2$ is determined, then $\mathrm{H}-3$ successively. As a result, the higher field peak at $4.4-4.6 \mathrm{ppm}$ and the peak at $2.72 \mathrm{ppm}$ correspond to $\mathrm{H}-1$ and $\mathrm{H}-2$ of GlcN residue, respectively. The resonance of $\mathrm{H}-3$ of GlcN residue is present at $3.54 \mathrm{ppm}$. On the other hand, the lower field peak at $4.4-4.6 \mathrm{ppm}$ corresponds to $\mathrm{H}-1$ of GlcNAc residue. The resonances of $\mathrm{H}-2$ and $\mathrm{H}-3$ of GlcNAc residue are present at 3.77 and $3.48 \mathrm{ppm}$, respectively. No attempts were made to identify the resonances of $\mathrm{H}-4, \mathrm{H}-5$, and $\mathrm{H}-6$, which occur in the region 3.3-4.1 ppm, because the spectra of them are more difficult to interpret. ${ }^{1} \mathrm{H}$ NMR spectrum was measured in the

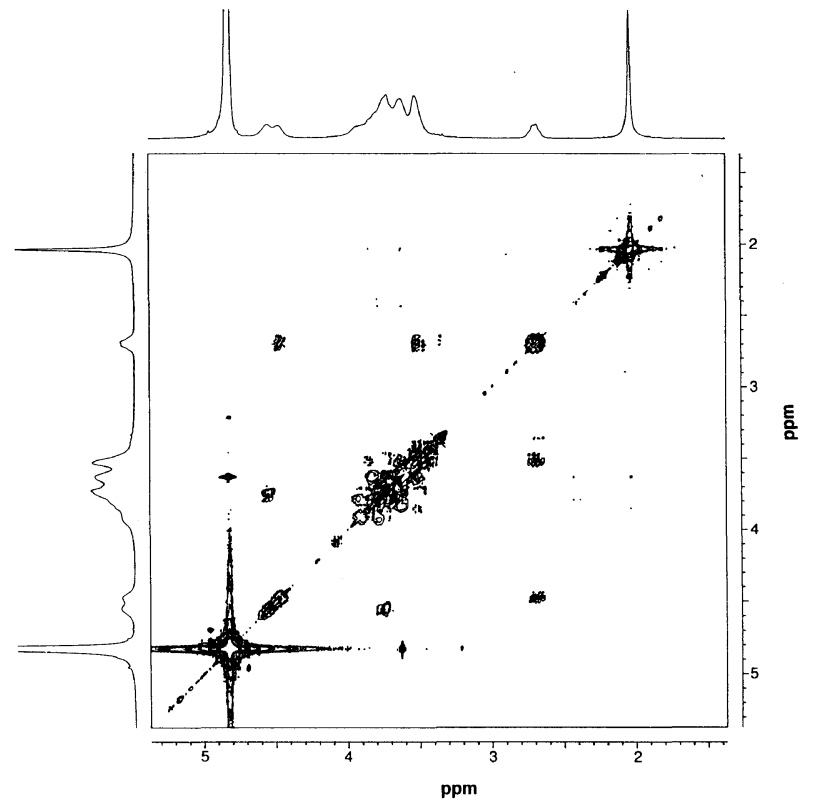

Figure 4. ${ }^{1} \mathrm{H}-{ }^{1} \mathrm{H}$ NMR correlations on partially $N$-acetylated chitosan in $\mathrm{D}_{2} \mathrm{O}$. The sample was derived from Ch-19.

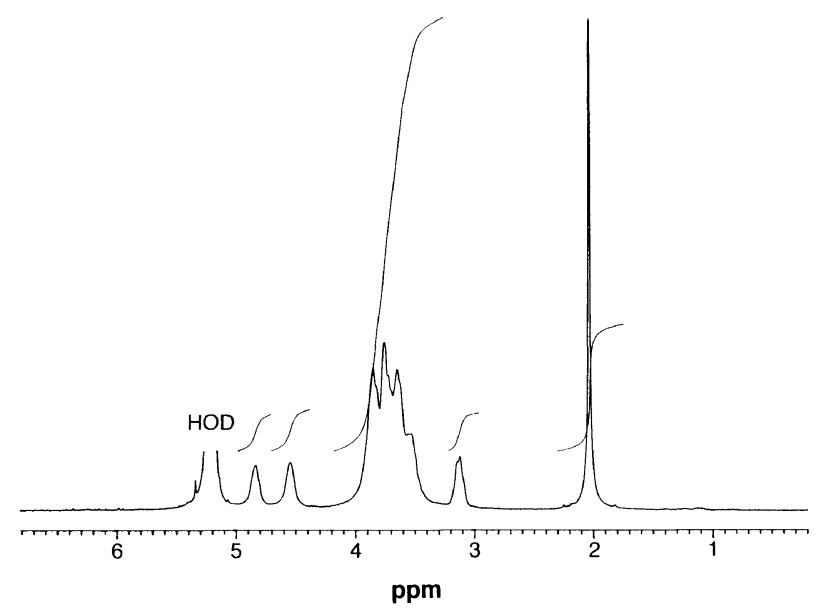

Figure 5. ${ }^{1} \mathrm{H}$ NMR spectrum of partially $N$-acetylated chitosan in $\mathrm{D}_{2} \mathrm{O}$ containing $\mathrm{CF}_{3} \mathrm{COOD}$. The sample was derived from $\mathrm{Ch}-19$.

presence of $\mathrm{CF}_{3} \mathrm{COOD}$ as well (Figure 5). Although acid has a deleterious effect on the polysaccharide backbone, it does not change the nitrogen substitution level. The assignments of signals were also confirmed by 2D NMR homonuclear chemical shift correlation maps (Figure 6). The comparison of ${ }^{1} \mathrm{H}$ NMR spectra of Figures 3 and 5 shows the significant downfield shift of $\mathrm{H}-1, \mathrm{H}-2$, and $\mathrm{H}-3$ of GlcN residue in the presence of $\mathrm{CF}_{3} \mathrm{COOD}$. For a purpose of this paper, the chemical shift data for assigned ${ }^{1} \mathrm{H}$ signals are listed in Table II.

Integral intensity ratio of $\mathrm{H}-1$ protons of $\mathrm{GlcN}$ and GlcNAc residues must represent the degree of $\mathrm{N}$ acetylation. Those signals, however, are too small, and we calculated the degree of $N$-acetylation, DA, from the following equation according to the report by Hirai et al. ${ }^{14}$

$$
\mathrm{DA}(\%)=\frac{I_{\mathrm{Ac}} / 3}{I_{\mathrm{H}-2,3,4,5,6} / 6} \times 100
$$

where $I_{\mathrm{Ac}}$ is the integral intensity of $N$-acetyl protons and $I_{\mathrm{H}-2,3,4,5,6}$ is the sum of integral intensities of $\mathrm{H}-2, \mathrm{H}-3, \mathrm{H}-4, \mathrm{H}-5, \mathrm{H}-6$, and $\mathrm{H}-6^{6}$ protons. A series of 


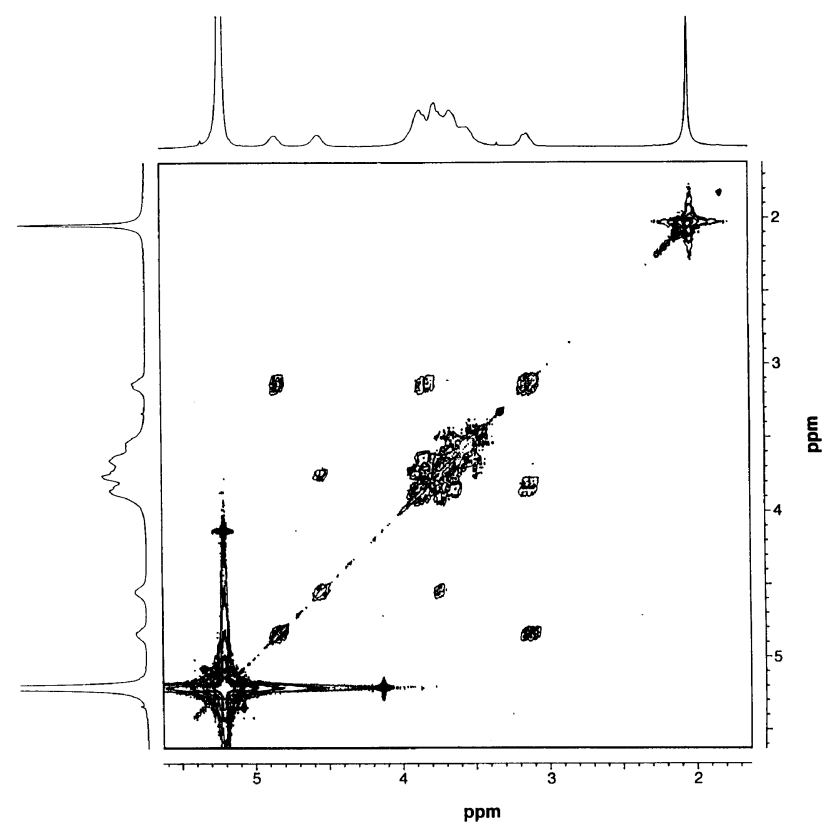

Figure 6. ${ }^{1} \mathrm{H}^{-1} \mathrm{H}$ NMR correlations on partially $N$-acetylated chitosan in $\mathrm{D}_{2} \mathrm{O}$ containing $\mathrm{CF}_{3} \mathrm{COOD}$. The sample was derived from Ch-19.

Table II. Assignment of ${ }^{1} \mathrm{H}$ NMR chemical shifts ${ }^{\mathrm{a}}$ of partially $N$-acetylated chitosan in $\mathrm{D}_{2} \mathrm{O}$ and $\mathrm{D}_{2} \mathrm{O}$ containing $\mathrm{CF}_{3} \mathrm{COOD}$

\begin{tabular}{|c|c|c|c|c|c|c|}
\hline Solvent & Residue & $\mathrm{H}-1$ & $\mathrm{H}-2$ & H-3 & $\mathrm{H}-4,5,6$ & $N$-Acetyl \\
\hline \multirow[t]{2}{*}{$\mathrm{D}_{2} \mathrm{O}$} & GlcN & 4.49 & 2.72 & 3.54 & -4.1 & - \\
\hline & GlcNAc & 4.56 & 3.77 & 3.48 & 4.1 & 2.04 \\
\hline \multirow[t]{2}{*}{$\mathrm{CF}_{3} \mathrm{COOD} / \mathrm{D}_{2} \mathrm{O}$} & GlcN & 4.86 & 3.16 & 3.87 & $3.4-4.1$ & - \\
\hline & GlcNAc & 4.57 & 3.77 & 3.52 & $3.4-4.1$ & 2.05 \\
\hline
\end{tabular}

${ }^{a}$ TMS was used as external reference.

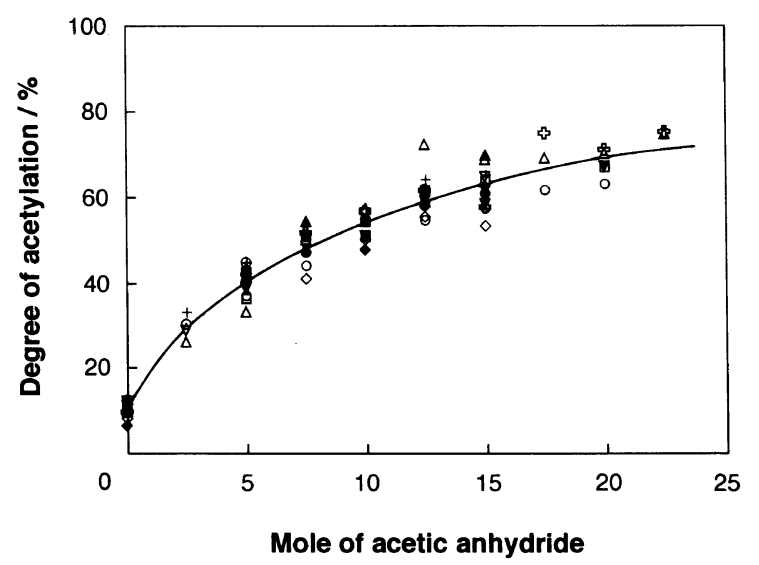

Figure 7. Percentage of $\mathrm{N}$-acetylation degree versus mole ratio of acetic anhydride to glucosamine unit: $\diamond, \mathrm{Ch}-8.8 ; \diamond, \mathrm{Ch}-12 ; \bigcirc, \mathrm{Ch}-19$; ๑, Ch-33; $\nabla$, Ch-63; , Ch-78; $\odot$, Ch-110; $\square$, Ch-140; $\square$, Ch-150; + , Ch-230; \&, Ch-340; $\Delta$, Ch-380; $\triangle$, Ch-600.

samples with different $N$-acetylation degrees were obtained by varying the amount of acetic anhydride. Figure 7 demonstrates the $N$-acetylation degree versus mole ratio of acetic anhydride to glucosamine unit available. As the molar amount of acetic anhydride increases, the degree of $N$-acetylation increases and gradually levels off, regardless of the molecular weight. An excess amount of acetic anhydride was necessary, since aqueous solution was used as solvent. These data were supported by elemental analysis, which indicated a

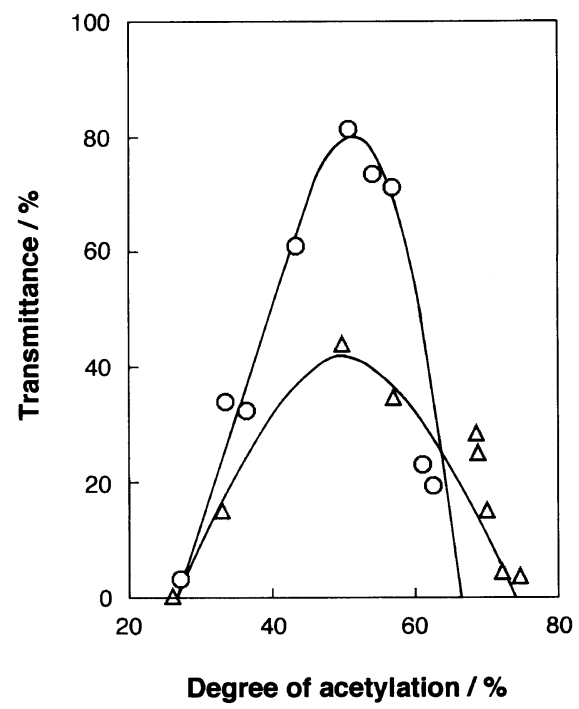

Figure 8. Dependence of water-solubility of chitosan on the degree of $N$-acetylation: $O, \mathrm{Ch}-19 ; \triangle, \mathrm{Ch}-600$.

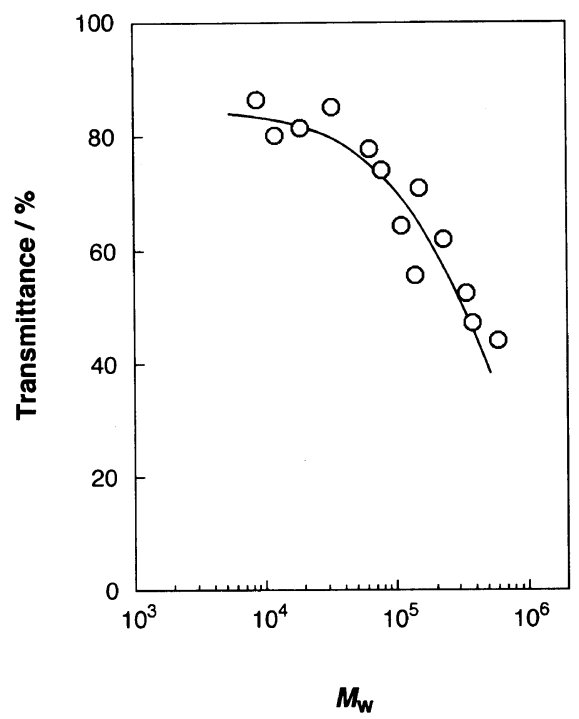

Figure 9. Dependence of water-solubility of $N$-acetylated chitosan on the molecular weight. Each sample has the $N$-acetylation degree of about $50 \%$.

comparable decrease in the molar percentage of nitrogen.

Water-solubility of the partially $\mathrm{N}$-acetylated derivatives obtained were evaluated in terms of turbidity. Figures 8 and 9 show the dependence of transmittance of the aqueous solution of $N$-acetylated chitosan on the degree of $N$-acetylation and on the molecular weight, respectively. $N$-acetylated chitosan with about $50 \%$ acetylation degree shows the highest water-solubility as expected and remains in solution. The range in the $\mathrm{N}$-acetylation degree of water-soluble derivatives is considerably narrow, and other samples with under $40 \%$ and over $60 \% \mathrm{~N}$-acetylation degrees fail to give clear aqueous solutions. With 8 to 10 -fold excess acetic anhydride, about $50 \%$ of amino groups can be acetylated (Figure 7). Moreover, it is apparent that water-solubility is influenced by the molecular weight as well as the degree of $\mathrm{N}$-acetylation; lowering the molecular weight helps to improve solubility. Because it was reported that blockwise distribution of $N$-acetyl groups reduced the water-solubility, ${ }^{8}$ the sequence of GlcNAc and GlcN 


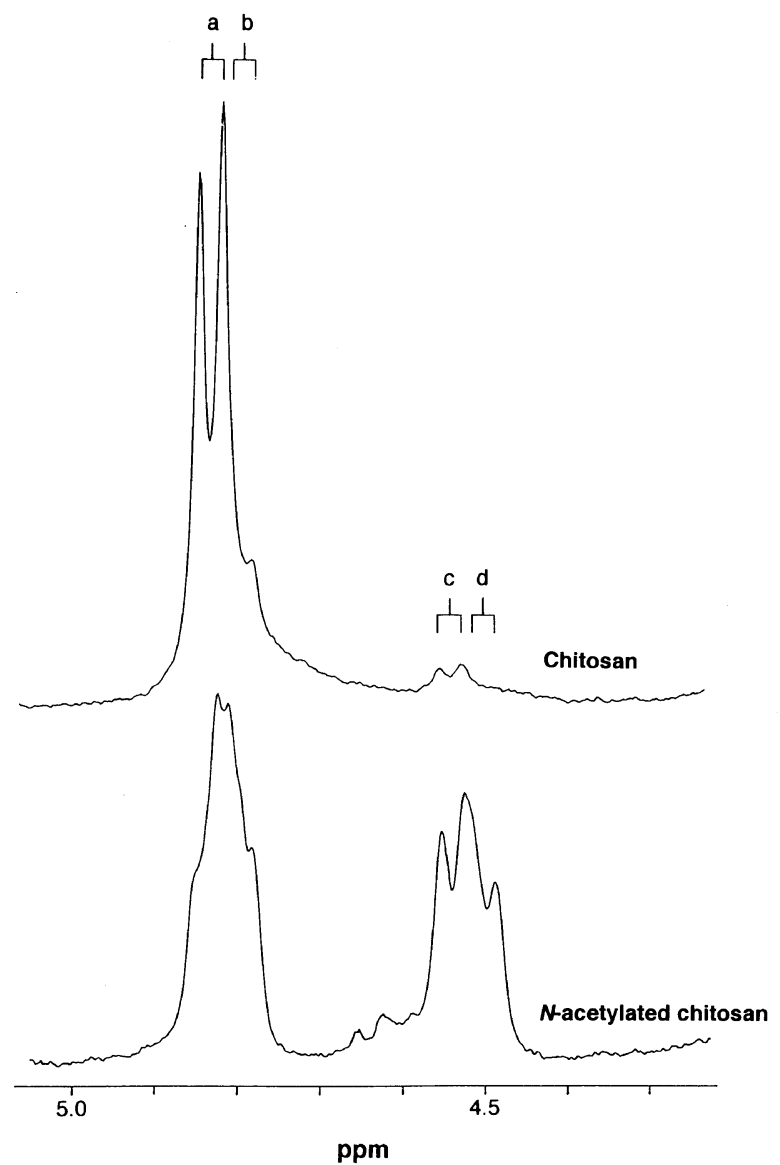

Figure 10. $\quad{ }^{1} \mathrm{H}$ NMR spectra in the region for resonances of anomeric protons of original chitosan and $N$-acetylated chitosan in $\mathrm{D}_{2} \mathrm{O}$ containing DCl at $80^{\circ} \mathrm{C}: \mathrm{a}, \mathrm{GlcN}-\mathrm{GlcN} ; \mathrm{b}, \mathrm{GlcN}-\mathrm{GlcNAc} ; \mathrm{c}$, GlcNAcGlcN; d, GlcNAc-GlcNAc.

residues was then investigated by ${ }^{1} \mathrm{H}$ NMR spectroscopy.

A sequence-dependent deshielding of $\mathrm{H}-1$ signals made it possible to determine the proportions of the four possible diads: GlcN-GlcN, GlcN-GlcNAc, GlcNAcGlcN and GlcNAc-GlcNAc. ${ }^{15}$ Figure 10 displays the region for the resonances of anomeric protons of original chitosan and partially $N$-acetylated chitosan derivatives in the presence of $\mathrm{DCl}$ at $80^{\circ} \mathrm{C}$. The resonance of $\mathrm{H}-1$ of GlcNAc residue shows two doublet peaks, c and d, that reflect two diads, GlcNAc-GlcN and GlcNAcGlcNAc, respectively. This result is supported by the fact that the GlcNAc-GlcN diad preponderates at lower $\mathrm{N}$-acetylation degree, i.e., original chitosan. Although Varun $e t$ al. reported two singlet peaks (or broad peaks) for these two diads, ${ }^{15}$ the H-1 must show a $J_{\mathrm{HH}}$ coupling with $\mathrm{H}-2$ and give a doublet peak as reported. ${ }^{12,16}$ The resonances of $\mathrm{H}-1$ of $\mathrm{GlcN}$ residue also contain information of the sequences: GlcN-GlcN (a) and $\mathrm{GlcN}_{-}$ GlcNAc (b). These findings suggest that the $\mathrm{H}-\overline{1}$ resonances are very sensitive to the nearest neighbor, most probably to the following residue in the chain. The mole fraction of GlcNAc-GlcN sequence, $F_{\text {GlcNAc-GlcN }}$, was estimated using the following equations ${ }^{15}$ :

$$
\begin{aligned}
& F_{\mathrm{GlcNAc}}+F_{\mathrm{GlcN}}=1 \\
& F_{\mathrm{GlcNAc}}=F_{\mathrm{GlcNAc}-\mathrm{GlcNAc}}+F_{\mathrm{GlcNAc}-\mathrm{GlcN}} \\
& F_{\mathrm{GlcN}}=F_{\underline{\mathrm{GlcN}-G l c N A c}}+F_{\mathrm{GlcN}-\mathrm{GlcN}} \\
& F_{\underline{\mathrm{GlcNAc}}-\mathrm{GlcN}}=F_{\underline{\mathrm{GlcN}-G l c N A c}}
\end{aligned}
$$

Polym. J., Vol. 29, No. 2, 1997
Table III. Distribution of diad frequencies of partially $\mathrm{N}$-acetylated chitosan as determined by ${ }^{1} \mathrm{H}$ NMR spectroscopy

\begin{tabular}{lcccc}
\hline \multirow{2}{*}{$\begin{array}{c}\text { Sample } \\
\text { code }\end{array}$} & $\begin{array}{c}\text { Degree of } \\
N \text {-acetylation }\end{array}$ & $F_{\text {GlcNAc-GlcNAc }}$ & $\begin{array}{c}F_{\text {GlcNAc-GlcN }} \\
\left(F_{\text {GlcN-GleNAc }}\right)\end{array}$ & $F_{\text {GlcN-GlcN }}$ \\
\cline { 2 - 2 } & $\%$ & & & \\
\hline Ch-8.8 & 53 & 0.27 & 0.26 & 0.21 \\
Ch-12 & 48 & 0.20 & 0.28 & 0.24 \\
Ch-19 & 51 & 0.24 & 0.27 & 0.22 \\
Ch-33 & 47 & 0.20 & 0.27 & 0.26 \\
Ch-63 & 48 & 0.24 & 0.24 & 0.28 \\
Ch-78 & 51 & 0.25 & 0.26 & 0.23 \\
Ch-110 & 51 & 0.23 & 0.27 & 0.22 \\
Ch-140 & 51 & 0.26 & 0.25 & 0.25 \\
Ch-150 & 50 & 0.24 & 0.26 & 0.24 \\
Ch-230 & 52 & 0.26 & 0.26 & 0.22 \\
Ch-340 & 52 & 0.26 & 0.25 & 0.23 \\
Ch-380 & 54 & 0.28 & 0.26 & 0.20 \\
Ch-600 & 50 & 0.24 & 0.26 & 0.24 \\
\hline
\end{tabular}

${ }^{a}$ Sample codes correspond to those in Table I.

Table III lists the diad frequencies for water-soluble chitosan derivatives with about $50 \%$ acetylation degree. The deviation from a random distribution is small: $24-28 \%$ for the GlcNAc-GlcN (or GlcN-GlcNAc) sequence. ${ }^{1} \mathrm{H}$ NMR characterization demonstrates that the $N$-acetylated chitosan derivatives have random $N$-acetyl moieties, regardless of the molecular weight.

In conclusion, it was revealed that chitosan could be rendered water-soluble by $N$-acetylation with acetic anhydride in simple aqueous acetic acid as solvent. The ${ }^{1} \mathrm{H}$ NMR spectra indicated that the sequence of GlcNAc and GlcN residues was random regardless of the molecular weight. The water-solubility of $N$-acetylated chitosan with about $50 \%$ acetylation degree showed the highest water-solubility, and the water-solubility increased as the molecular weight decreased.

\section{REFERENCES}

1. E. R. Pariser and D. P. Lombardi, "Chitin Sourcebook," Wiley, New York, N.Y., 1989.

2. N. Kubota, Bull. Chem. Soc. Jpn., 66, 1807 (1993).

3. N. Kubota, Y. Kikuchi, Y. Mizuhara, T. Ishihara, and Y. Takita, J. Appl. Polym. Sci., 50, 1665 (1993).

4. N. Kubota, E. Kai, and Y. Eguchi, Maku, 21, 386 (1996).

5. M. Yalpani, "Chitin in Nature and Technology," R. A. A Muzzarelli, C. Jeuniaux, and G. W. Gooday, Ed., Plenum, New York, N.Y., 1986, p 403.

6. H. Struszczyk, J. Appl. Polym. Sci., 33, 177 (1987).

7. T. Sannan, K. Kurita, and Y. Iwakura, Makromol. Chem., 177, 3589 (1976).

8. K. Kurita, T. Sannan, and Y. Iwakura, Makromol. Chem., 178, 3197 (1977).

9. K. Kurita, Y. Koyoma, S. Nishimura, and M. Kamiya, Chem. Lett., 1597 (1989).

10. S. Aiba, Int. J. Biol. Macromol., 11, 249 (1989).

11. Y. Kawamura, H. Ikebe, I. Kurahashi, H. Seo, and S. Nakajima, Kokai Tokkyo Koho, 61-40337 (1986).

12. M. Rinaudo, P. Le Dung, C. Gey, and M. Milas, Int. J. Biol. Macromol., 14, 122 (1992).

13. K. Kurita, S. Chikaoka, M. Kamiya, and Y. Koyama, Bull. Chem. Soc. Jpn., 61, 927 (1988).

14. A. Hirai, H. Odani, and A. Nakajima, Polym. Bull., 26, 87 (1991).

15. K. M. Varum, M. W. Anthonsen, H. Grasdalen, and O. Smidsrod, Carbohydr. Res., 211, 17 (1991).

16. A. Domard, C. Gey, M. Rinaudo, and C. Terrassin, Int. J. Biol. Macromol., 9, 233 (1987). 